\title{
Design and development of a new biomedical/open surgical instrument
}

\section{Zheng Li}

University of Bridgeport, Bridgeport, USA

Email: zhengli@bridgeport.edu

Received 19 May 2009; revised 30 May 2009; accepted 1 June 2009.

\section{ABSTRACT}

This article introduces a new biomedical / open surgical instrument to assist surgeon in applying surgical clips to patient's body tissue and blood vessel during surgical processes. The new clip delivery system is designed to better the clip's distal advance through internal clip channel, jaw guiding track, and all other transition areas to keep surgical clip from accidental shooting out during clip's distal move into jaws. Currently the clip distal move in normal surgical instrument is usually driven by compression springs and some complains of clip accidental drop-off were recorded in surgical procedures. Because higher request of dimensional tolerance and better component surface quality are needed in case the compression springs are used as driven force, a little dimensional deviation or less qualified part surface produced from manufacturing processes will potentially cause surgical clip device malfunction or misfiring of the clips. It is clearly known that the jaws can seriously sever or damage patient's blood vessel or body tissue if there is no clip inside the jaws due to accidental clip drop-off, when surgeons close instrument handles. The improved internal system design in this new open surgical instrument can prevent clip from accidental drop-off because of well guided and controlled clip distal move through internal clip channel and track. Besides the operational force to fully form clip is lower than existing surgical clip devices due to better mechanical advantage in this new instrument design. In addition to the above, manufacturing and product cost can be decreased since lower requirement of dimensional tolerance and surface quality of instrumental parts is allowed in this new surgical instrument design. This new instrumental prototype is build upon the analysis of computer aided modeling and simulation to prove its good mechanical advantage, feasible function, reliable performance. The preliminary results of instrument firing force from both computer aided modeling and prototype testing are very close to each other, and preliminary prototype testing shows no accidental clip drop-off in this new biomedical / surgical clip instrument.

Keywords: Blood Vessel; Body Tissue; Ligation; Computational Modeling; Mechanical Advantage

\section{INTRODUCTION}

It is critical for surgeons to apply surgical clip instrument to the severed organs, blood vessels and body tissues to stop bleeding in surgical procedure $[1,2,3]$. The surgical clip instrument jaws should be put around patent blood vessel or body tissue and clips can be closed to secure the vessel and tissue to prevent them from bleeding when surgeon bring instrument handles together. Next surgical clip will be automatically driven into instrument jaws through internal clip channel when surgeons release instrument handles.

The surgical devices to apply clips onto blood vessel and body tissue have been developed for many years $[4$, $5,6]$. It includes single clip and multiple clip applications $[7,8,9]$. Next clip can be loaded into instrument after firing each clip in single clip applications and multiple clips can be sequentially applied to the vessel or tissue in multiple clip applications. The surgical clip devices usually have two handles, a master body, a clip loading and crimping assembly, clip driving mechanism and some other functioning components such as clip pusher and jaws. The improvement for better clip advancement and lower cost instrument is continued to develop more reliable, better functional and cost-effective clip instruments to support surgeons in their surgical processes.

Some complains were previously recorded from clinic fields showing several incidents of accidental clip shooting out from jaws while clip was driven into jaws 
in clip instruments using compression springs as driving force. These incidents showed that the closed jaws will potentially sever or damage blood vessel or body tissue if there is no clip in jaws. The accidental clip drop-off is mainly caused by improper control of dimensional tolerance when manufacture the instrumental components. The surgical instruments in which surgical clips were driven by compression spring require higher dimensional tolerance control and better surface quality during component manufacturing and this will lead a low volume and high cost instrumental production, otherwise the clips will drop-off or shooting out if dimensional tolerance are too loose or clips will jam or not move by spring force if dimensions are too tight.

This new instrument design has different clip delivery system compared with current clip instruments. The distal movement of clip pusher driven by instrument handles gradually advances clip from clip magazine, through clip channel and transition area between clip channel and jaws, and finally form clip after clip being driven into guide track in jaws. The clip pusher returns proximally to its original start position when surgeons release instrument handles and then readily picks up next clip. Since the internal clip delivery can be easily and well controlled through simple lever-linkage driving system in this new design, the accurate dimensional tolerance control and higher surface quality are not required during production and manufacturing processes of instrumental components. This can lead cost-effective machining process, increase production volume, and reduce production cost. The preliminary prototype testing also indicates that there is no accidental clip shooting out in this new instrument design and operational force to fully form clip is lower than current surgical clip instruments.

This new instrumental prototype testing has been conducted on dogs including vascular occlusion, ligating for tubular ducts, and applying surgical clip to animal tissue. In vitro and in vivo studies were conducted to investigate the clip holding force, degradation rate, and tissue reactivity for clips of titanium material. The clips were applied across excised canine cystic ducts by this new instrument and both axial and transverse pull-off forces have been recorded and measured. In the next phase, the titanium clips were implanted subcutaneously into the animals and the remaining strength within the clips has been measured after $1,3,5,7,9,11,13$, or 21 days. The preliminary results show that the clips applied by this new surgical instrument function properly.

The preliminary testing results also indicated that there is no clip shooting out in this surgical instrument design and the operating force to fully form the clip is between $3.16 \mathrm{lbf}$ and $3.30 \mathrm{lbf}$ which are lower than $4 \mathrm{lbf}$ in existing surgical instrument.

\section{ANALYSIS OF NEW SURGICAL CLIP INSTRUMENT}

The prototype, instrument front and rear portion views of this new surgical instrument are indicated in Figure 1-3. First the instrument will be put around patient blood vessel or body tissue. When surgeons start and gradually close instrument handles, clip driven by clip pusher advances distally from clip magazine through internal track and channel into jaws and finally secured blood vessel or body tissue. The instrument jaws can be open as surgeons release instrument handles, and clip pusher and driving bar return to its original start positions. Compared with current instrument that clip delivery is driven by compression spring, the clip being loaded into jaws is well guided and controlled in this new instrument design. The driving bar linked to instrument handles at pivot point moves distally to drive clip pusher that advances clip steadily into jaws as surgeons gradually close surgical instrument handles. Such a stable clip linear motion in this new design can be easily and well controlled by surgeons to prevent clip from accidentally shooting out from instrument. The preliminary prototype testing of

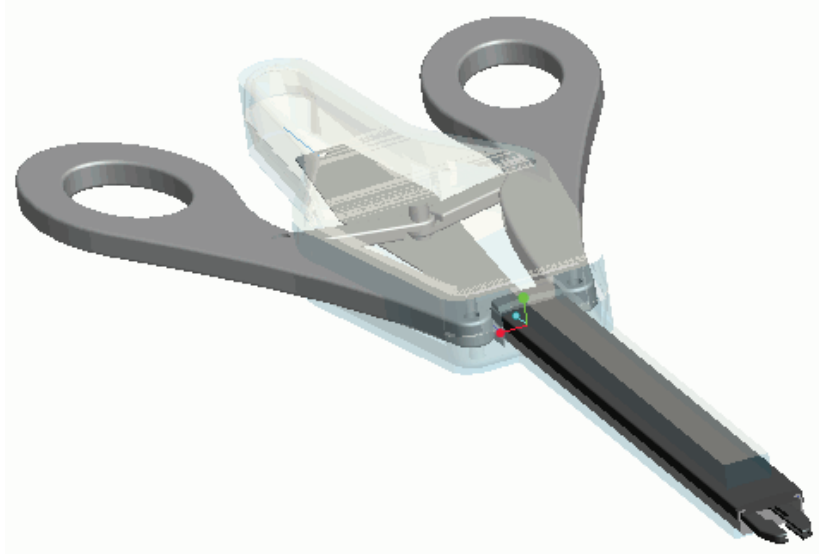

Figure 1. Prototype of new surgical instrument.

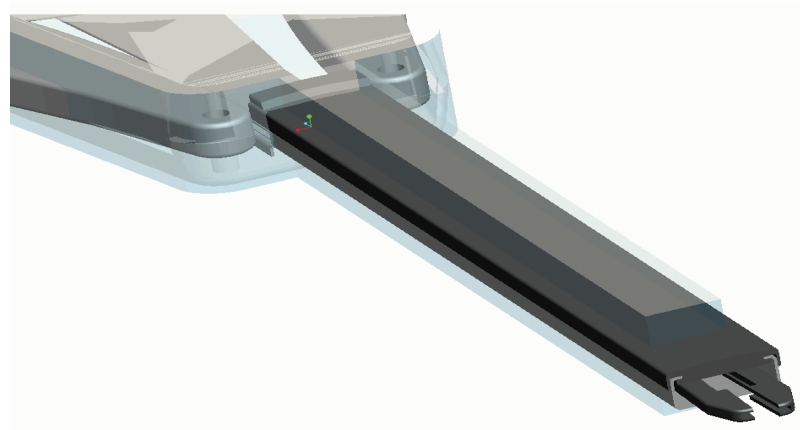

Figure 2. Front portion view of new surgical instrument. 


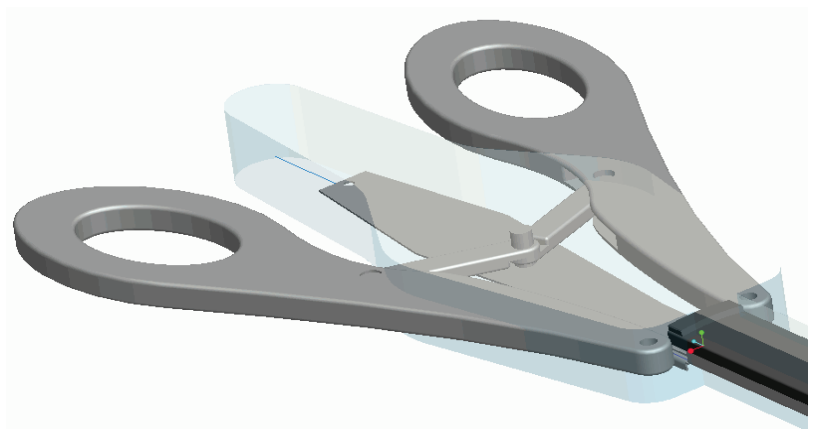

Figure 3. Rear portion view of new surgical instrument.

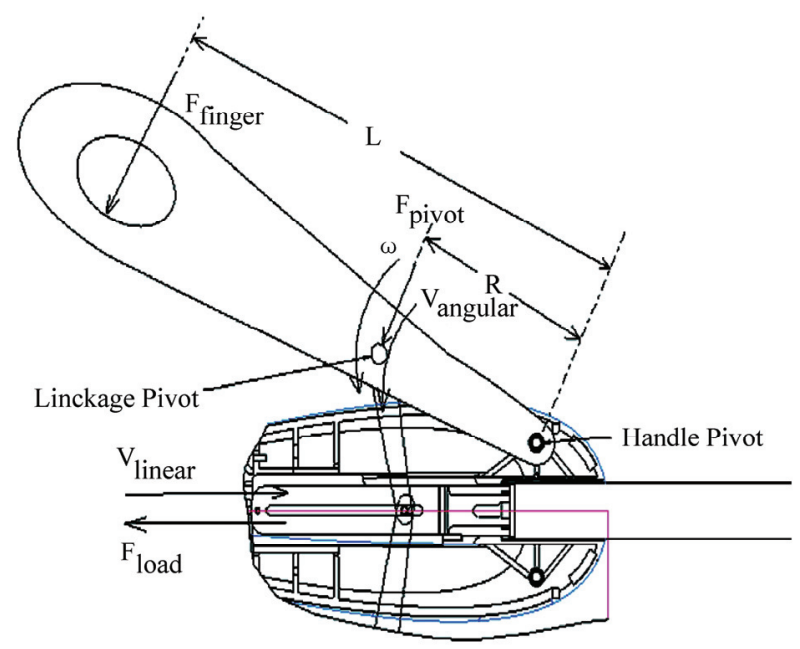

Figure 4. Force analysis diagram in instrument.

this new design indicated its proper and reliable performances with no clip shooting out and lower operational force than usual to form surgical clips.

\section{COMPUTATIONAL MODELING AND ANALYSIS}

Referring Figure 4, the energy balance and force equations in this new surgical instrument design can be derived as follows.

$$
\mathrm{F}_{\text {load }} * \mathrm{~V}_{\text {linear }}=\mathrm{T} * \omega
$$

Because torque $\mathrm{T}=\mathrm{F}_{\text {pivot }} * \mathrm{R}$,

$$
\mathrm{T} * \omega=\mathrm{F}_{\text {pivot }} * \mathrm{R} * \omega=\mathrm{F}_{\text {pivot }} * \mathrm{~V}_{\text {angular }}
$$

Then

$$
\begin{gathered}
\mathrm{F}_{\text {load }} * \mathrm{~V}_{\text {linear }}=\mathrm{F}_{\text {pivot }} * \mathrm{~V}_{\text {angular }} \\
\mathrm{F}_{\text {load }}=\left(\mathrm{V}_{\text {angular }} / \mathrm{V}_{\text {linear }}\right) * \mathrm{~F}_{\text {pivot }}=(\mathrm{VR}) * \mathrm{~F}_{\text {pivot }}
\end{gathered}
$$

Referring the force diagram of this instrument handle in Figure 4:

$$
\mathrm{F}_{\text {finger }} * \mathrm{~L}=\mathrm{F}_{\text {pivot }} * \mathrm{R}
$$

The different $F_{\text {finger }}$ can be determined with different combination of $\mathrm{L}$ and $\mathrm{R}$. The computational simulation can find the optimized values of $\mathrm{L}$ and $\mathrm{R}$ to reduce the operational force to fully form clip. The computer aided solution suggested $\mathrm{L}=4.85$ inch and $\mathrm{R}=2.20$ inch for best instrument performance.

Based on Eq.5,

$$
\begin{gathered}
\mathrm{F}_{\text {finger }} * 4.85=\mathrm{F}_{\text {pivot }} * 2.20 \\
\mathrm{~F}_{\text {pivot }}=2.20 * \mathrm{~F}_{\text {finger }}
\end{gathered}
$$

Referring Eq.4,

$$
\mathrm{F}_{\text {load }}=(\mathrm{VR}) * \mathrm{~F}_{\text {pivot }}=(\mathrm{VR}) * 2.20 * \mathrm{~F}_{\text {finger }}
$$

The velocity ratio of $\left(\mathrm{V}_{\text {angular }} / \mathrm{V}_{\text {linear }}\right)$ can be determined by computer aided simulation targeting optimized instrument performance, and simulation results are shown in Figure 5.

The mechanical advantage of this new instrument can be found when surgical clip is fully formed:

$$
\begin{aligned}
& \text { Mechanical advantage }=(\mathrm{VR}) * 2.20 \\
& =(.04940 / .03548) * .20=3.063
\end{aligned}
$$

This result shows that, if $20 \mathrm{lbf}$ forces are required to fully form the surgical clip, the operational force that surgeon needed is $3.265 \mathrm{lbf}$ that is lower than normal spec of $4 \mathrm{lbf}$ and this will benefit surgeons in their surgical procedure. Also, both computational simulation and prototype testing results are very close which verify and prove the credibility of this new instrument design and research methodology.

\section{CONCLUSIONS}

The prototype of this new surgical clip instrument has been proved to have feasible function and better performance based on instrumental functional study, computer aided modeling and solution, and preliminary prototype testing. This new instrument design has several

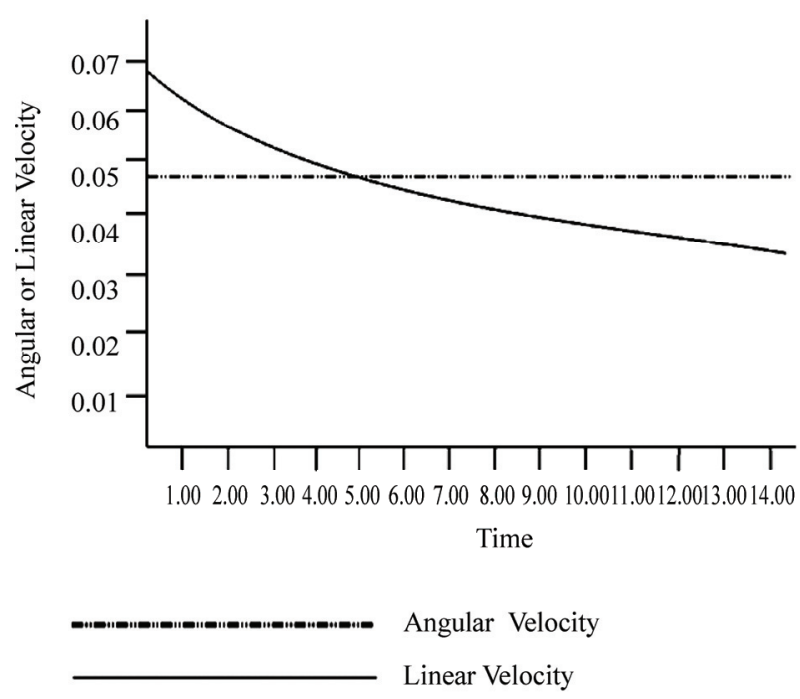

Figure 5. Linear and angular velocity vs. time phase in operating the instrument. 
major advantages compared with some current surgical clip devices including: the clip's distal move can be properly guided and controlled to prevent patient's blood vessel and body tissue from damage that caused by accidental clip shooting out during surgical processes, operational force to form clip is lower than usual surgical clip instruments, instrument production and product cost can be decreased since loose dimensional tolerance and low surface quality of instrumental components are allowed in this new design. The prototype testing has been conducted in lab and the preliminary result has shown the potential improvement over some current instruments including reliable performance without accidental clip drop-off, reduced operational force to form clip, and lower product cost. While the prototype this new instrument is being sent to fields for more clinical trials and evaluations, further analysis and improvement will be conducted including adding support to enhance jaw pair structure to prevent jaws from twisting when unexpected torque load exerted to the instrument due to improper usage in surgical procedure, adding enhancing structure to prevent jaws from accidental close when unanticipated side load exerted to instrument jaws, simplifying the instrument design to further reduce the product cost.

\section{REFERENCES}

[1] H. J. Lin, W. C. Lo, Y. C. Cheng, and C. L. Peng, (2007) Endoscopic hemoclip versus triclip placement in patients with high risk peptic ulcer bleeding, Journal of Gastroenterol, 102, 539-543.
[2] D. Chan, J. Bishoff, I. Ratner, L. Kavoussi, and T. Jarrett, (2000) Endovascular gastrointestinal stapler device malfunction during laparoscopic nephrectomy: Early recognition and management, Journal of Urology, 164(2), 319-321

[3] J. Hermiller, C. Simonton, T. Hinohara, and D. Lee, (2005) Clinical experience with a circumferential clipbased vascular closure device in diagnostic catheterization, Journal of Invasive Cardiology, 17, 154-157.

[4] J. Piatt, B. Starly, E. Faerber, and W. Sun, (2006) Application of computer-aided design methods in craniofacial reconstructive surgery using a commercial image-guidance system, Journal of Neurosurgery, 104(1 Suppl), 64-67.

[5] A. W. Cheng, P. W. Chiu, P. C. Chan, and S. H. Lam, (2004) Endoscopic hemostasis for bleeding gastric strormal tumors by application of hemoclip, Journal of Laparoendoscopic \& Advanced Surgical Techniques, 14, 169-171.

[6] B. Starly, Z. Fang, W. Sun, and W. Regli, (2005) Three-dimensional reconstruction for medical-CAD modeling, Journal of Computer-Aided Design and Application, 2(1-4), 431-438.

[7] P. Evans, B. Starly, and W. Sun, (2006) Computer-aided tissue engineering for design and evaluation of lumbarspine arthroplasty, Journal of Computer-Aided Design and Application, 3(6), 771-778.

[8] W. Sun, B. Starly, J. Nam, and A. Darling, (2005) Bio-CAD modeling and its application in computer-aided tissue engineering, Computer-Aided Design, 37(11), 1097-1114.

[9] F. C. Chu and B. C. Chang, (2005) Automatic visual tracking control system using embedded computers, Proceeding of the 2005 IEEE International Conference on Mechatronics, July 10-12, 2005.

$\mathrm{F}_{\text {finger }}-\mathrm{lbf}$, load on surgeon's finger

$\mathrm{V}_{\text {linear }}$ - inch per second, linear distal moving velocity of instrument drive bar

(VR) - velocity ratio

$\mathrm{R}$ - inch, distance between handle pivot and linkage pivot 